\title{
The Use of Locative Expressions in Dependence of the Spatial Relation between Target and Reference Object in Two-Dimensional Layouts*
}

\author{
Hubert D. Zimmer ${ }^{1}$, Harry R. Speiser ${ }^{1}$, Jörg Baus ${ }^{2}$, \\ Anselm Blocher $^{3}$, Eva Stopp ${ }^{3}$ \\ ${ }^{1}$ Department of Psychology, University of Saarland \\ PO Box 151150, D- 66041 Saarbrücken \\ huzimmer@rz.uni-sb.de; h.speiser@rz.uni-sb.de \\ ${ }^{2}$ SFB 378, University of Saarland \\ PO Box 151150, D- 66041 Saarbrücken \\ baus@cs.uni-sb.de \\ ${ }^{3}$ Department of Artificial Intelligence, University of Saarland \\ PO Box 151150, D- 66041 Saarbrücken \\ blocher@cs.uni-sb.de; stopp@cs.uni-sb.de
}

\begin{abstract}
In two experiments we investigated the use of German locative expressions as a function of the spatial relation between a reference object (RO) and a to-be-located object (LO). In the experiments, a speaker described to another participant, by locative expressions, where LO can be found in relation to RO. LO (a blue dot) was presented at different positions around RO (a red dot). The listener saw RO only, and her or his task was to find LO by moving a small window over the screen using the computer mouse. The positions of LO were circularly arranged around RO and their angular relations were varied in steps of 15 degrees. In Experiment 1, only the four canonical expressions (left/right, above/below) and their single composites were allowed. In Experiment 2, no constraints were made. Both experiments yielded comparable results. The canonical expressions were used nearly exclusively for prototypical relations, and their production latencies were the shortest. Composites were used for all non-prototypical relations. There was only a small spatial area next to the canonical directions in which two different locative expressions were used, and in these areas of competition the longest production times were observed. Thus, canonical expressions were used in a much smaller area around the prototypical axes than predicted by selection rules based on applicability ratings obtained in meta-linguistic judgments.
\end{abstract}

\footnotetext{
* This research was supported by a grant from the German Research Foundation in the Special Research Division SFB 378 'Resource-Adaptive Cognitive Processes'.
} 


\section{Introduction}

Imagine a situation in which someone is standing in front of a city map which is mounted at the wall of a tourist office. She or he is searching for a target position on this map, e.g., the TV tower. The actual position is highlighted as a red spot which can be easily found. However, dependent on the density of the depicted information, it might be hard to find the intended location. The visitor might ask another person for help, and this person might answer: "The TV tower is to the right of the red spot, which indicates your present position". This is the task we wanted to simulate in the experiments.

We are interested in the use of projective locative expressions. More precisely speaking, we are interested in the use of such expressions depending on the angular spatial relation between the to-be-located object and a reference. We investigated the use of the four canonical locative expressions in German - 'unten' [below], 'oben' [above], 'links' [on the left], 'rechts' [on the right] - and their single composites 'links oben', [on the left and above], 'rechts oben' [on the right and above], 'links unten' [on the left and below] and 'rechts unten' [on the right and below].

Projective locative expressions are comprised of three components: a to-be-located object, a reference object, and a directional spatial expression, e.g., 'on the left of', 'above', etc. (Herskovits 1985, Logan 1995). In the case of a person wanting to describe a location using verbal means, the speaker must explicitly encode spatial relations into verbal expressions. We assume that people have a non-verbal spatial representation of a perceived layout, and that they map this non-verbal representation onto a verbal one. During the verbal encoding process, two basic problems have to be solved: a reference system has to be specified, and a locative expression has to be selected to indicate the target position within this reference system. We assume, that for this purpose, the speaker projects a dimensional coordinate system onto the spatial layout, and dependent on the angular relation between RO and LO a verbal expression is then selected which specifies the intended region within these coordinates.

Furthermore, we assume that the origin of the coordinate system is located in the reference point, and that the orientation of the coordinates is given by the perspective taken by the speaker and adopted by the listener (cf., Herrmann, 1996). The point of view can either be suggested by the reference object itself (the intrinsic case) or by an external point of observation (the perspective of the speaker or the listener) (cf., Grabowski, 1996; Levelt 1989; Retz-Schmidt 1988). In the example, the reference point will most likely be the highlighted position (the red spot), and the speaker and the listener share their point of view, i.e. they behave as if they were standing side by side facing the map.

The use of a specific coordinate system depends on the cultural habits (Klein, 1994). Members of the Western culture most likely use a frame of reference with three canonical axes: up-down, left-right, and front-back (e.g., Bryant, Tversky \& Franklin, 1992). Under certain conditions, one of these axes can be ignored, so that the system is reduced to two dimensions. In the example, it is reduced to a vertical plane. Projective locative expressions label spatial areas in the direction of the four 
base axes, e.g., 'to the right of' or 'above'. However, these expressions are ambiguous in respect to the spatial area which they indicate. For instance, 'to the right of' can correspond (a) to the right half plane, (b) to a more or less extended sector to the right of the reference object, and (c) to a position that is on the right axis or in a narrow spatial area next to it (cf. Herskovits, 1985, p. 181). A position on the right diagonal in a horizontal plane, for example, is therefore equally acceptable as being 'to the right' as well as being 'in front of' the reference position (cf. Franklin, Henkel \& Zangas, 1995). In other words, the spatial expressions are not mutually exclusive. Consequently, there is no one to one correspondence between a spatial location and an expression, forcing the speaker to select among a set of spatial expressions which are correct in a logical sense.

In order to solve this problem, we have to define a selection mechanism or a rule to select the expression. For this purpose, we can use the construct of applicability. The applicability of an expression is the degree to which a real spatial relation fits the ideal or prototypical condition which would represent the meaning of that expression in the best way. The ideal meaning of a projective expression is defined by a focal area which holds a specific spatial angular relation to the reference object. For example, "'to the right' is an instance of a graded concept with the right axis as its focal region" Herskovits (1986, p. 184). By this definition for each spatial expression, a field of decreasing applicability is defined with it's maximum at the prototypical spatial area. The speaker might then select the expression which has the highest applicability for the spatial region, and if more than one expression is left, the most specific one might be used (cf. Levelt (1989) for a similar general conception of the selection of a lexical entry).

Based on these theoretical assumptions, the applicability of a projective spatial expression (and also its selection) should primarily be dependent on the deviation of the angular direction in reality from the prototypical angular direction. Empirical support for this relationship was reported by Gapp (1995). He presented his subjects with configurations of two simple geometric objects with different angular relations between a to-be-located object and a reference object, and he asked his subjects to judge the applicability of different expressions from 0 to 1 (from not applicable to perfectly applicable). He observed that the ratings decreased with increasing angular deviations from the prototypical positions. The same result was reported by Hayward \& Tarr (1995) who also investigated the applicability of projective spatial expressions (left, right, above, below) dependent on the angular direction between RO and LO. Due to these results Gapp (1997) used the applicability as a means to select the best fitting spatial expression in a verbal localization task, in which a computer automatically generated spatial descriptions. Similarly, Logan \& Sadler (1996) suggested a spatial template rule which was also based on the idea of a varying degree of applicability. These authors subdivided the spatial area in three fields of applicability to describe the use of the four locative expressions. The prototypical direction (good), the 'not acceptable' area (a deviation of $90^{\circ}$ and more) and the acceptable region (the area between the prototypical and the not acceptable area).

However, although these studies revealed very consistent data, they have the weakness of being based on metalinguistic judgments regarding language use, and 
this behavior does not necessarily correspond to a real production situation. Subjects rated the applicability of a given spatial expression to describe a specific angular relation between LO and RO. This is a listener's perspective. The evaluation process, from this perspective might differ from the conditions in which the real language output is generated. Metalinguistic judgments might therefore not reflect the true selection conditions. It is also possible that the ratings reflect rational arguments which give the presented examples a post-hoc plausibility. In the real production situation the observed language output might have been shown for a different reason. There exits empirical data supporting this assumption. Franklin, Henkel \& Zangas (1995), for example, observed that subjects used more often 'left' and 'right' than 'in front of' which had been expected from the metalinguistic judgments, and Hayward \& Tarr (1995) reported that the use of 'left and 'right' was restricted to the area of prototypical use, although these expressions were accepted as applicable in a much wider area.

These data provide evidence that it is necessary to investigate the use of these spatial expressions, not only by judgments, but also in overt production. In order to do this, we simulated, in the laboratory, a production situation in which a speaker had to describe a spatial relation between LO and RO to a listener who only knew the position of RO. We introduced the listener to make the production conditions more real and to set up a situation in which locative expressions had a real pragmatic function. In addition, we systematically varied the spatial relation between RO and LO. We did this the same way it was done in the study from Gapp (1995) in which the applicability was rated.

\section{Experiment 1}

In the experiment, the production of locative expressions was investigated under conditions which simulated everyday situations. A speaker informed another person, using locative expressions about the position of an object which was being searched for. Therefore, the subjects took part in the experiment in pairs. One subject served as speaker, and the other as listener.

Subjects produced spatial descriptions to a visual display presenting two small dots, one served as RO and one as LO. LO was placed in different locations circularly arranged around RO. The speaker was instructed to describe where LO could be found by using one of eight spatial expressions: the four canonical expressions ('links', 'rechts', 'oben' 'unten') or one of the four single composites, e.g., 'links oben'. We tape-recorded the produced descriptions so that we could count the frequencies of usage, and we compared it with the frequencies which were expected due to the selection rules based on the applicability ratings of the same eight expressions. In addition, we measured the production latencies (speech-onset-time), by a voice key, as an indicator of the difficulties of selection. The task of the recipient was to find the hidden LO on the computer screen. Using a computer mouse, the listener moved a small window over the display. The LO was then made visible as soon as the window hit the target. 


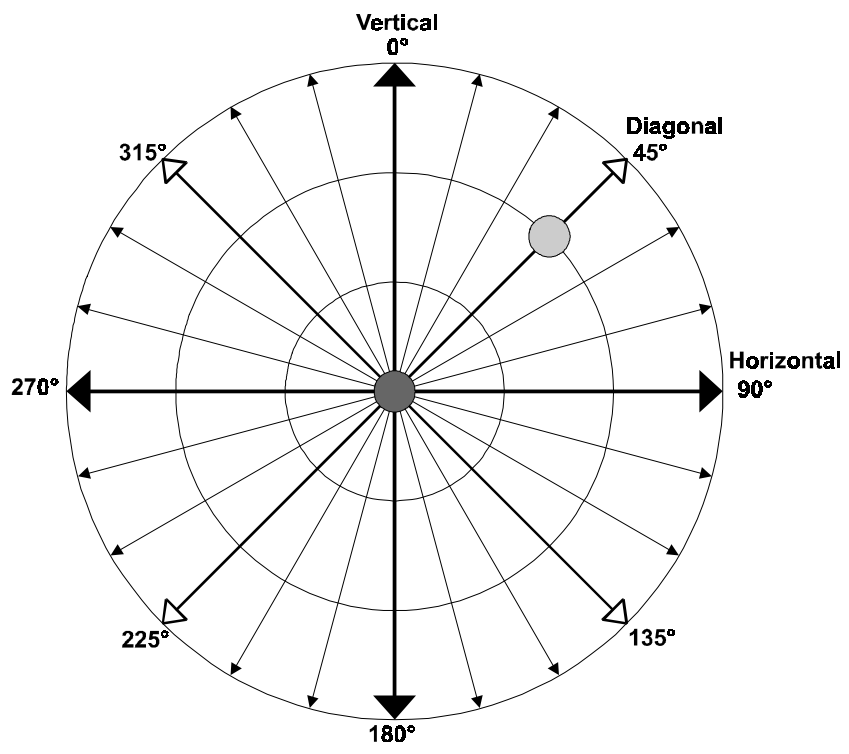

Fig. 1. An illustration of the angular relations obtained in the experiments. LO (the light gray dot) could be presented in each of the 24 directions around RO (the dark gray dot). The lines are for illustration. During the experiment only RO and LO were visible

As an independent variable, we varied the angular relations between LO and RO (cf. Figure 1). We expected that the observed frequencies of expressions and the production latencies were a function of the angular relations between LO and RO. If the use of a spatial expression depends on the deviation of the actual angular relation between LO and RO from the prototypical relation of the expression, we should find that the production frequencies decrease with an increase of deviation from the ideal area of use. In addition, if the selection rules which were based on metalinguistic judgments, can be used for productions (Gapp, 1997), the frequency of using a spatial expression should follow the rating of applicability. Gapp computed the applicability of composite relations in such a way that their applicability decreased faster with angular deviation than the applicability of the canonical locative expressions. Consequently, the sector, in which composite expressions are used, should be smaller than the one for canonical expressions. Composite expressions should therefore be less frequent than canonical expressions.

For the production latencies similar assumptions can be made. It is plausible to predict that the shortest latencies should occur when the angular relation has the best fit to template. The latencies should become longer the more the conditions deviate from the optimal angular relation. The spatial expressions for canonical directions 
$\left(0^{\circ}, 90^{\circ}, 180^{\circ}, 270^{\circ}\right)$ should therefore be produced very quickly. If corresponding spatial templates for composite expressions exist, these expressions should behave in the same manner. The diagonal directions $\left(45^{\circ}, 135^{\circ}, 225^{\circ}, 315^{\circ}\right.$, cf. Figure 1$)$ should be named quickly. All other relations which are non-prototypical should be processed more slowly.

However, regarding the production latencies we have to consider a further influence. The selection model discussed so far, is based on the assumption that the 'mental space' is as homogeneous as the physical space. All things being equal, comparing the different directions no anisotropy should exist, and as a consequence, the only factor which influences the selection of a spatial expression is the deviation from the prototypical direction. Contrary to this, data from psychological experiments suggest that the accessibility of spatial information varies with orientation. If subjects mentally accessed an object in a specific location, the above-below direction was easier to evaluate than the ahead-behind direction which was again easier than the left-right direction (Franklin \& Tversky, 1990; Logan, 1995). It is therefore possible that the production latencies are influenced by two factors. One is the deviation from the prototypical direction, and the other is the absolute direction in which LO is located.

In addition to these production data, we also measured the search time of the listener from the initiation of the mouse movement up to the moment when the window touched the dot. This time was taken as an indicator for the appropriateness of the used expression. The better the locative expression indicated a specific position, the easier it should have been for the listener to find the dot, therefore shortening the search time.

\subsection{Method}

Subjects. Thirty students of the University of Saarland took part in the experiment. All subjects were native German speakers and were paid for their participation. All factors were manipulated within subjects.

Material and Design. The experiments were controlled by two HP workstations with a PC connection. The configurations for the speaker and the listener were presented on the workstations' 17' computer screens. The PC measured the verbal response latencies using a soundcard which was utilized as a voice key.

As 'objects' a blue and a red dot were presented, which were solid circles with a diameter of $1 \mathrm{~cm}$. Circles were chosen because they do not favor a specific orientation of the coordinate system. The red dot was defined as reference object and the blue dot was defined as the to-be-located object.

The reference object was randomly displayed at the corner of an invisible square which was centered in the middle of the screen. This variation should help avoid that subjects use absolute screen positions instead of relative positions, and it should make plausible to use projective expressions. 
The to-be-located object was presented on 72 different positions which laid on 24 directions around the RO. Figure 1 illustrates the possible positions. The angles between these lines were $15^{\circ}$. The set of relations consisted of the four canonical directions $\left(0^{\circ}, 90^{\circ}, 180^{\circ}, 270^{\circ}\right)$, the four diagonal directions $\left(45^{\circ}, 135^{\circ}, 225^{\circ}, 315^{\circ}\right)$, and the non-prototypical directions in between. The directions which were prototypical for a spatial expression are highlighted by bold lines in Figure 1. On each line three different positions were defined to allow for variable distance between RO and $\mathrm{LO}(3.50 \mathrm{~cm}, 5.25 \mathrm{~cm}$, and $7.00 \mathrm{~cm})$.

Procedure. Subjects were run in pairs. One subject served as speaker, the second subject, the listener, had to find, as quickly as possible, the hidden target on the screen. After the completion of one phase, i.e., all 72 relations were presented, the roles were exchanged. The 72 trials, i.e., different angular relations, were presented in random order.

Subjects were seated in front of their computers. Their stations were separated by a folding screen so that they could talk, but not see each other. At the beginning, both subjects could practice in both roles to familiarize themselves with the complete task. All subjects reported to be experienced in using a computer mouse.

Each trial had the following structure. A short warning signal (a beep) was presented. One second later, the speaker saw RO (the red dot), and $250 \mathrm{~ms}$ after stimulus onset LO (the blue dot) was presented. The speaker was instructed to say aloud a locative expression that indicated the spatial position of the target, as quickly as possible. As spatial expressions 'unten' [below], 'oben' [above], 'links' [on the left]', 'rechts' [on the right] and their single composites 'links oben' [upper left], 'rechts oben' [upper right], 'links unten' [lower left] and 'rechts unten' [lower right] were allowed. Subjects were free to choose which way they sequentially arranged the locative expressions within the composites. They could use, for example, 'rechts oben' or 'oben rechts'. - A later analysis revealed that in nearly all cases, subjects started with the horizontal dimension first. - The speaker was instructed to verbalize only the spatial expressions and not to formulate whole sentences. We tape recorded the verbalizations for a later analysis of the used expressions. This procedure should have minimized the contribution of additional sentence planning processes to the production latencies which were outside the scope of our research. Subjects were neither allowed to verbalize absolute positions (e.g., in the top right-hand corner of the screen) nor to specify the distances between RO and LO.

The speaker's production triggered a voice key. This impulse released the presentation of the listener's reference object surrounded by a square the size of the dot. This square served as a search window. It was attached to the mouse pointer, so that the move of the window corresponded the movement of the mouse in speed and direction. The search window was first bound to the position of RO, and it could be unlocked by a mouse click. As soon as the window touched the invisible target, the dot was displayed. A further mouse click finished the current trial and started the next one. 


\subsection{Results}

The frequencies of the used expressions, the production latencies, the comprehension times, and the search times were analyzed. Overall there were only a few invalid trials, $3.4 \%$ were caused by the speaker, and $2.8 \%$ by the recipient. The production latency was defined as the time from the presentation of RO up to the speech onset which triggered the voice key. The comprehension time was estimated using the time from the initiation of the speech up to the first mouse click by the listener. However, because the comprehension times strongly correlated with the production latencies we have not reported these data because we could not exclude the possibility that the comprehension times were influenced by the time the speaker needed to utter the expressions. The search time was measured using the time between this mouse click and the contact of the search window with the invisible dot. The order of runs, i.e. whether the subject first had the role of a speaker or of a recipient, did not influence the results. We therefore excluded this factor from all analyses.
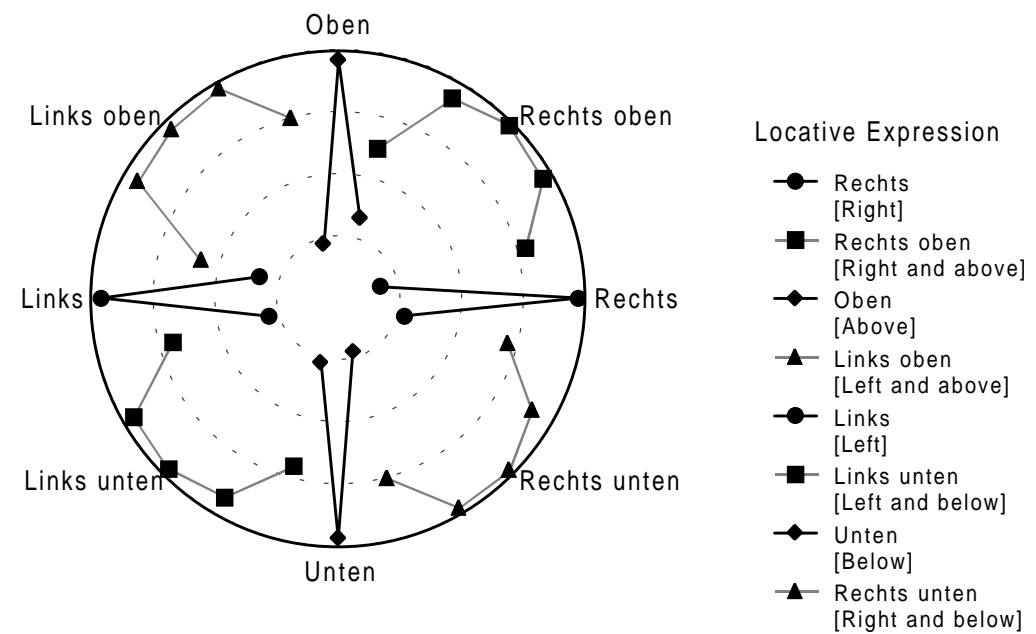

Fig. 2. Frequencies of use of the different spatial expressions (in percentages) as a function of the angular relation between the to-be-located object and the reference object in Experiment 1. The radius of the circle indicates the maximum possible value ( $0 \%$ to $100 \%$ ); the concentric dotted circles indicate steps of $25 \%$. The spatial relations are the same as in Figure 1

First, we analyzed the used spatial expressions. For each expression, the average frequency of its use was computed dependent on the spatial relation between RO and 
LO. In Figure 2 these results are illustrated as percentages of use dependent of the spatial relation between LO and RO. Since subjects did not produce wrong descriptions, only the proportions of prototypical and composite expressions are given. Frequencies less than $10 \%$ are not shown.

Clearly, subjects used the canonical locative expressions only in the exact prototypical case, i.e. the four main axes. All diagonal and nearly all non-prototypical relations were labeled with composite expressions, and the data were symmetric around these axes. Therefore, for further analyses, the naming frequencies were combined in three classes of expressions each of which corresponded to one prototypical area: the vertical direction (above/below), the horizontal direction (left/right) and the two diagonal directions (single composites). Geometrically, this procedure is equivalent to a projection of all conditions into the upper right-hand quadrant. Figure 3 illustrates the average frequencies of the three types of expressions dependent on the angular deviation from the prototypical area.

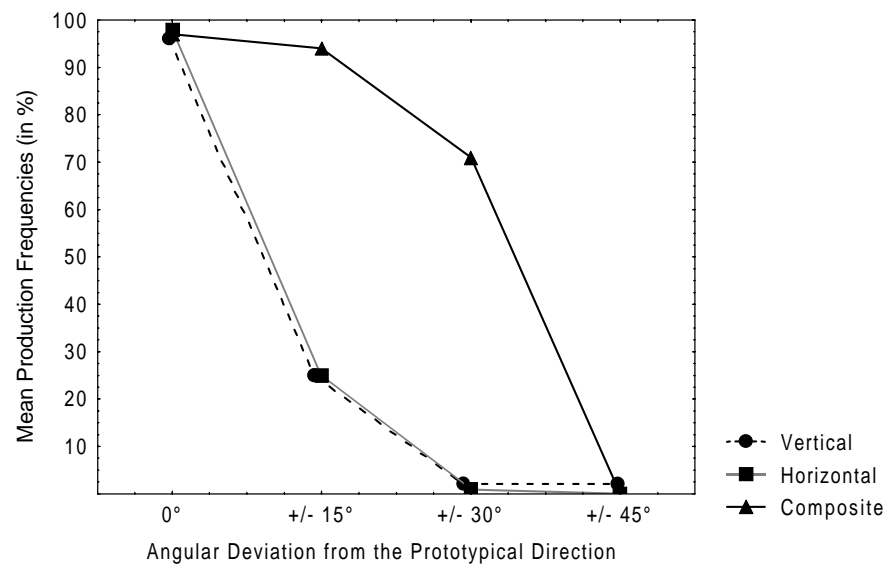

Fig. 3. Frequencies of use of vertical, horizontal and composite expressions (in percentages) as a function of the angular deviation of the to-be-located object from the prototypical direction

As expected, the frequencies of production decreased with the increase of the angular deviation from the prototypical direction. However, the shape of this function is different for the three classes of expressions. A comparison of the vertical and horizontal dimension showed no difference, $\left(\chi^{2}(3)=4.3, p>.20\right)$, but both were different from the diagonal dimension, $\left(\chi^{2}(3)=71.1, p<.001\right)$. This is reflected by the different slopes of the three frequency curves over angular deviation. At its prototypical area, each of the three 'correct' expressions was used nearly exclusively. However, if the spatial relation deviates only by $15^{\circ}$ from this area, for canonical expressions, the curve declined abruptly to zero, whereas the decrease for the composite expressions started at stronger deviations. At $15^{\circ}$ deviation, composites were produced as frequently (94\%) as at the prototypical area (97\%), whereas at the 
same deviation the canonical relations already dropped to a very low frequency of usage $(25 \%)$.

Similarly to the frequencies, the production latencies were analyzed. A difficulty with voice key measures is that the point at which the stopwatch is triggered, depends on the amplitude of the speech signal which is partially dependent on the phoneme at the word's beginning (Pechmann, Reetz \& Zerbst, 1989). The latencies of different locative expressions which start with different phonemes, e.g., 'links' and 'rechts', are therefore not necessarily comparable. Based on this information, we ran a control experiment to test whether such differences would influence our production latencies. In this experiment, subjects had only to read aloud the visually presented locative expressions, and we measured the production latencies. No significant differences between the two sets of locative expressions starting with 'links' [left] and 'rechts' [right] were found $(F(1,9)<1)$. However, the vertical canonical descriptions ('oben'/'unten') [above/below] were produced about $100 \mathrm{~ms}$ more quickly than all other expressions, $\mathrm{F}(1,9)=19.6, \mathrm{p}<.01$. We should therefore be careful when interpreting effects of this dimension in the production latencies of the main experiment. More important, however, is that the speech onset of all other expressions were comparable.

Due to these results in the control experiment we next analyzed the production latencies as a function of the angular relation between LO and RO. In a first step, the mean latencies for the production were calculated independent of the used expressions. These data are depicted in Figure 4.

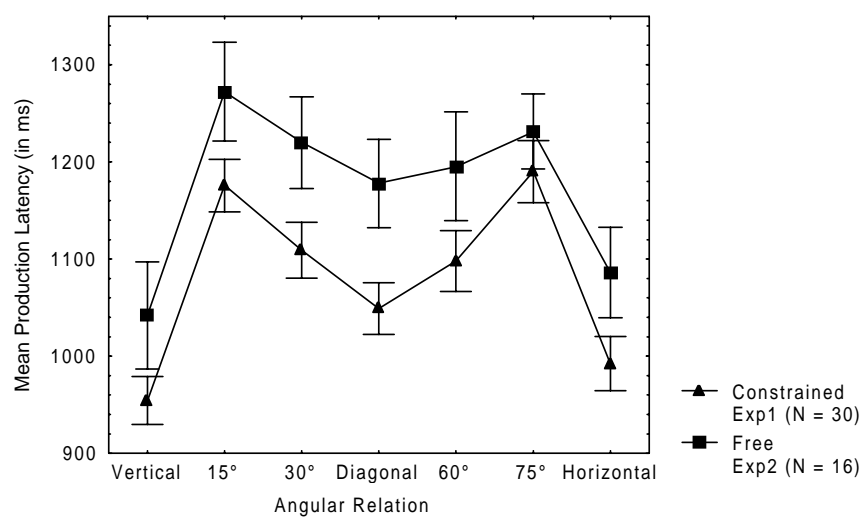

Fig. 4. Average production latencies of the spatial expressions (in ms) as a function of the angular relation between the to-be-located object and the reference point in Experiment 1 (set of locative expressions was pre-specified) and 2 (free production). All relations are projected into the upper right quadrant of the complete circular layout. The error bars indicate plus minus one standard error

In a first analysis, we compared the latencies for the three classes of expressions (vertical, horizontal, and diagonal) in the prototypical directions, i.e., when only the 
typical expressions were used. Latencies increased from vertical to horizontal to diagonal dimensions, $F(2,58)=7.5 ; p<.01, M S E=9748(946 \mathrm{~ms}, 994 \mathrm{~ms}$, and 1044 ms, respectively).

In a second analysis, the latencies for the diagonal directions were analyzed with the additional factor angular deviation $\left( \pm 0^{\circ}, \pm 15^{\circ}\right)$. The main effect of angular deviation was significant, $F(1,29)=6.2 ; p<.02, M S E=5448$. The latencies increased from the exact direction $(1045 \mathrm{~ms})$ to the $\pm 15^{\circ}$ condition $(1097 \mathrm{~ms})$. In other words, with a stronger deviation from the prototypical direction, longer production latencies were observed. A more extreme deviation $\left( \pm 30^{\circ}\right)$ produced even longer production latencies (1189 ms). However, this effect cannot be accepted as support of the angular deviation hypothesis. The longer production latencies are probably not due to the stronger angular deviation from the prototypical area, but are more likely dependent on the fact that subjects had to select between two different expressions. This is shown in the following analysis.

In an over all analysis, independent of the used expression, the spatial relations which were next to the canonical dimensions showed the longest production latencies of all conditions, $F(1,29)=104.9, M S E=8276, p<.001$. It should be again noted, that this was the only condition in which different expressions were used. It is therefore likely that, in this case, two expressions are in competition to each other. This assumption is supported by a final analysis in which we compared the production latencies for canonical expressions and composites, both at their prototypical angular relations and at the conditions with competition (cf. Fig. 5).

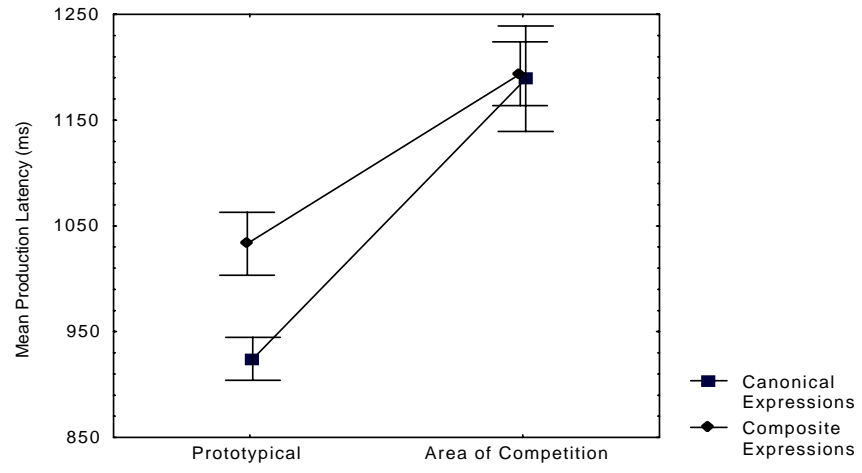

Fig. 5. Production latencies for canonical and composite locative expressions in their prototypical positions and in the area of competition in Experiment 1. The error bars indicate plus minus one standard error

This analysis was based on only 19 subjects because all subjects were excluded who did not use different kinds of locative expressions at the area of competition. The analysis revealed that in the prototypical case composites were produced more slowly than canonical expression, although both were equally adequate. In addition, 
canonical and composite expressions did not differ from each other in the conditions with competition, and in this case both expressions were produced more slowly than in the prototypical ones, $F(1,18)=78.6 ; p<.001, M S E=10948$. This does not support the assumption that the production times increase with the degree of angular deviation, because in the competition area canonical expressions only had an angular deviation of $15^{\circ}$ from their prototypical area, whereas composite expressions had a deviation of $30^{\circ}$ from their prototypical area.

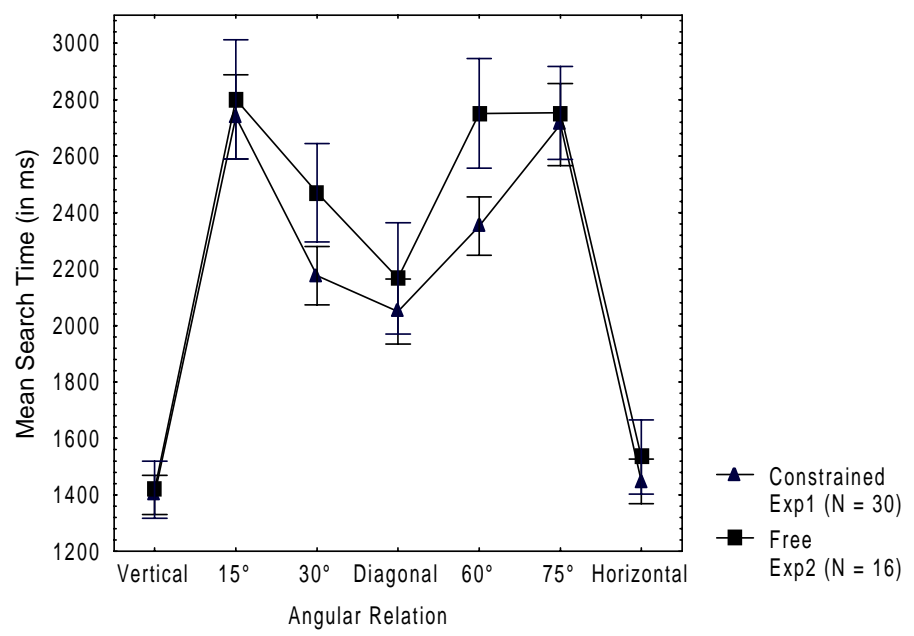

Fig. 6. Average search times (in ms) as a function of the angular relation between the to-belocated object and the reference point in Experiment 1 (set of expressions was pre-specified) and 2 (free production). All relations are projected into the upper right quadrant of the complete circular layout. The error bars indicate plus minus one standard error

Finally, we analyzed the search times (cf. Fig. 6). These times also varied over the angular relations between LO and RO, $F(6,174)=50.4, p<.001, M S E=174992$. The search times were shortest with the canonical relations. Objects in diagonal directions and at $\pm 15^{\circ}$ on both sides of it were discovered more slowly, but there was only a small increase from the prototypical to the $\pm 15^{\circ}$ condition. The longest search times were observed in those cases where LO was next to the canonical dimensions, i.e., the conditions with competition. Once more, we separated the two cases in which canonical expressions and composites were used. We also observed that the search times for the canonical directions $(1411 \mathrm{~ms})$ were faster than that of the prototypical diagonal directions $(1977 \mathrm{~ms})$. Subjects who had to locate an object in the competition area, found the targets faster if a canonical expression was used (2354 $\mathrm{ms}$ ) than if a composite expression was used $(3119 \mathrm{~ms})$. For the listener the canonical expression was more useful than the composite expression, although the speaker used the canonical expression less frequently than the composite. 


\section{Experiment 2}

Before we discuss these results, we want to report a second experiment. The data of Experiment 1 were clear-cut, however, they had one restriction. Subjects were instructed as to which set of locative expressions they should select their expression from. This was a necessary restriction in the experiment, due to our interest in the production conditions for the eight locative expressions. However, in order to be able to generalize the data to everyday production conditions, which do not have this restriction, it would be nice to have data from an experiment in which subjects were free to choose any locative expression.

In such an experiment we expected a higher variation of the used spatial expressions. This would make it impossible to analyze the complete data as a function of specific expressions and angular relations. However, if our data were at least partially replicated, we should observe the same results as in Experiment 1 for those subjects who used the locative expressions. We therefore expected fast productions of canonical expressions for prototypical spatial relations. In these cases, in Experiment 1 , canonical expressions were used almost exclusively, and this should remain so in a free production condition. With angular deviations from these axes, the variability of expressions might increase but if the results are comparable, with small angular deviations from the canonical axes again composites or other types of qualified expressions should be used. In addition, if different expressions are in competition to each other in the areas, on both sides of the canonical axes, the longest production times should be found. Furthermore, if subjects produce adequate descriptions of the spatial relations, the pattern of the search times over angular relations should not differ from those of Experiment 1.

\subsection{Method}

Subjects. Thirty students from the University of Saarland who were paid for their participation took part in the experiment. All subjects were native German speakers.

Material and Procedure. The material and the general procedure were the same as in Experiment 1. The only change in the procedure was that now subjects were free in their usage of locative expressions. During the instructions, the subjects were not directed as to which expression should or should not be used. The only remaining restriction was that only verbal descriptions were allowed, e.g., no pointing, etc.

\subsection{Results}

In this experiment the number of errors was again low. The speaker caused $6.1 \%$ of errors, and the recipient $4.3 \%$. 
As we expected, the variability of the used spatial descriptions increased. Half of the subjects used other reference systems rather than locative expressions. Ten used a reference system analogous to an analog clock, e.g., 'at two o'clock' or 'five to twelve'. Two subjects used circular degrees, two used a geographical reference system (north, east, south-west, etc.), and two subjects mixed different systems. The other 16 subjects used locative expressions. However, for these subjects the variability of expressions increased. In those cases, in Experiment 1 composite expressions were used, more complex qualifications were now more frequently given. Examples are: 'rechts und etwas oben' [to the right and a bit above], 'rechts diagonal oben' [to the right and diagonally above]', etc.

Due to this variability of the used complex locative expressions, we only reported the production and search latencies in dependence with the angular relation between LO and RO independent of the used expressions. Additionally, only those sixteen subjects, who used locative expressions, were further analyzed. For prototypical relations, these data reflect the latencies for canonical locative expressions, because these were nearly exclusively used with these directions. For all other angular relations, the latencies were averaged over all types of expressions. Therefore, these data integrated latencies over different types of expression specifying a canonical locative term. These data are depicted together with those of Experiment 1 in Figure 4 and 6 .

These data reveal that the production latencies as well as the search times showed a pattern that was similar to Experiment 1. The production latencies strongly varied with the angular relation between LO and RO $F(6,90)=11.5 ; p<.001, M S E=9389$. Canonical expressions, with prototypical positions were produced faster than all expressions at other spatial relations, $F(1,15)=29.4 ; p<.001, M S E=18786$. The longest production latencies were again observed at the area of competition, i.e. \pm 15 beside the canonical directions, $F(1,15)=33,7 ; p<.001, M S E=7856$. The differences between the exact diagonal and the intermediate spatial relations, however, were even smaller than in Experiment 1, and these latencies did not significantly differ from each other, although the advantage of the diagonal relation was numerically present.

If one compares the production latencies in the two experiments in a two way analysis of variance with Experiment as an additional factor, and Spatial relation (canonical or diagonal) as the second factor, the two main effects were significant. The production latencies increased from Experiment 1, constrained production: 1011 $\mathrm{ms}$, to Experiment 2, unconstrained production: $1121 \mathrm{~ms}, F(1,44)=6.2 ; p<.02$, $M S E=40407$. Furthermore, locative expressions were produced more quickly for canonical $(1005 \mathrm{~ms})$ than for diagonal relations $(1094 \mathrm{~ms}), F(1,44)=23.5 ; p<.001$, $M S E=7988$. Furthermore, locative expressions were produced more quickly for canonical $(1005 \mathrm{~ms})$ than for diagonal relations $(1094 \mathrm{~ms}), F(1,44)=23.5, M S E=$ $7988, p<.001$. Finally, the general pattern of the production latencies, as a function of the angular relation between $\mathrm{LO}$ and RO, was the same in both experiments $(F<1)$.

The same is true for the search times (cf. Fig. 6). The search times were shorter for the canonical directions than for all other spatial relations, $F(1,15)=148.9 ; p<.001$, $M S E=2848586$, and the times in the area of conflict were the longest, $F(1,15)=$ 
49.1; $p<.001, M S E=3506595$. The search times, in the diagonal direction, were longer than in the canonical direction but shorter than in the area of conflict. If one compares the search times in the two experiments neither the factor Experiment nor the interaction was significant.

\section{General Discussion}

The experiment should reveal how the use of locative expressions depends on the spatial relation between LO and RO, and how it depends on the deviation of LO's direction from the prototypical axes of the specific locative expressions. In addition, we wanted to test whether the actual usage of the locative expressions corresponded to the frequencies which were predicted on the basis of the applicability ratings. We got a clear answer to both questions. The use of locative expressions clearly depended on the angular relation between LO and RO but the frequencies were different from those which we expected from the applicability ratings.

In the prototypical regions of all expressions, we observed that in these ideal cases the 'correct' expression was used exclusively. However, the two classes of expressions showed different usage frequency patterns dependent on angular deviation when outside of the prototypical region. The canonical expressions were only used in their prototypical area. In all other directions composite relations were used which was even the case if LO was displaced from the prototypical axis by only $15^{\circ}$. Canonical relations therefore had a very small area of use, whereas composite relations had a wide area of application. From the selection rule, which was used by Gapp (1997), we had expected that composite expressions would have a narrow area of use, and canonical ones a large area of use, which was the opposite of the result.

Two possibilities exist to adjust the model to the observed data. One possibility is to find a different selection rule to modulate the observed data using the existent functions. The alternative is to use different applicability functions, possibly, in addition, with another selection rule. The applicability ratings, as metalinguistic judgments, might differ from the applicability values which are actually used in a production situation. This possibility is supported by the fact that also others reported deviations between the usage of locative expressions and the given ratings (e.g., Franklin, Henkel \& Zangas, 1995; Hayward \& Tarr, 1995). Perhaps this change could even be observed when subjects were required to judge the 'probability of use' and not the 'applicability of the expression'. Such a difference between the listener's view and the speaker's (functional) view is plausible, if one considers that 'what is acceptable' might differ from those things 'which are suitable or which are optimal to reach the communicative goal', i.e., a quick search for LO. Since locative expressions serve as means to reach the latter goal, one should consider this functional perspective as an alternative to the metalinguistic perspective when modulating the selection of locative expressions.

The production latencies followed a similar pattern as the frequencies. The productions of expressions at canonical directions were the fastest. It took longer to 
produce the composite expressions to diagonal relations, although these spatial relations matched the ideal direction as well as the horizontal and vertical relations matched the ideal spatial relations for canonical expressions. The longest production times were observed in the area of competition, in which both types of relations, i.e. composite and canonical expressions, were used. However, the production latencies did not directly follow the angular deviations. It looked more like an ordinal relationship. The verbalizations of the canonical relations were the fastest, followed by the composites which in a large sector around the diagonal direction were produced nearly at the same speed. In the area of competition, the longest production times were observed. This pattern of results is in good correspondence to the idea of a spatial template (cf. Logan \& Sadler, 1996), if one adds the assumption that composite locative expressions are used outside of the prototypical area of the canonical locative expressions.

Based on these results, we can speculate which cognitive processes caused these data. We assume, that two groups of processes are operating. One set specifies the canonical locative expressions. These processes are quickly finished as soon as the coordinate system is adjusted to RO. With the frame of reference, the prototypical directions for the four canonical locative expressions are given. For example, if LO is located "in a strip with axis the right base axis, and abutting on the reference object" (Herskovits, 1986, p.184) the locative expression 'to the right of' is immediately accessed. The same is true for the other half axes.

If the object is clearly not located in these positions, but in the diagonal sector, other processes are operating. Here we can think of two possibilities. One alternative is, combining two locative expressions that label the axes of the corresponding quadrant of LO. The two expressions should already be activated, because the application of a reference system specifies the applicability of canonical directions, i.e., the half planes bounded by a coordinate axis. These expressions are then combined to a composite expression. In this case, no additional evaluation processes for the applicability of diagonal relations are necessary, but only an additional process which combines the canonical terms.

Another alternative is to treat composites as primary expressions. In this case, a further process might be started which evaluates the exact diagonal direction as the prototypical relation for composites. In support of the latter assumption, the small advantage of the exact diagonal compared to the intermediate positions can be mentioned. However, this might not be a general effect. It might only occur in constrained situations, as in Experiment 1. There, the composite expressions were explicitly pre-specified as the alternatives to be used. In Experiment 2, no expressions were mentioned. In this unconstrained condition, the advantage of the exact diagonal direction was clearly reduced. The latter effect speaks in favor of the first alternative.

Finally, we have to assume the existence of a third component: an additional mutual inhibition of two possible expressions, followed by an active selection. These processes are necessary if two expressions are available, which compete with each other. In our experiments, this is the case at directions near to but not on the canonical axes. In this condition, one expression has to be selected, and vice versa the other one has to be inhibited. This additional processing slows down the production latencies by 
about $200 \mathrm{~ms}$. Such delays were also observed in other verbalization tasks in which two words compete with each other, and one has to be inhibited, whereas the other one has to be selected (e.g. Glaser \& Glaser, 1989). It is therefore very likely that a comparable selection process is effective at positions on either side of the canonical directions $\left( \pm 15^{\circ}\right)$. These directions are near enough to the area of application for canonical terms, so that these terms are activated but at the same time LO is also located within the 'diagonal area' that favors the use of a composite term. This exactly defines the competition.

A final word has to be said on the search times. Here too, the canonical conditions were the fastest. This could be expected because the areas of application for canonical expressions were small, and in correspondence, they clearly indicated a small area where the target could very likely be found. In contrast, the composite expressions had a wide area of use, and as a consequence they were less informative about the location. Subjects therefore had to search in a large area and which caused longer search times. The longest times were observed in the regions next to the canonical axes because they were last to be touched if subjects started to search from the diagonal which is the prototypical area for composite expressions. Due to this asymmetry of the areas of competition, the canonical expressions were more useful in terms of the search times, although they were used less often.

In summary, our data clearly support the idea that the use of locative expressions are a function of the deviation of the actual direction of LO from the prototypical directions of the respective expressions. Canonical expressions were used nearly exclusively for canonical relations, and they were uttered very quickly. In all other cases, composites were used which required more time. In addition, the areas of application were nearly non-overlapping, so that there was a clear correspondence between a spatial direction and the used expression. Only with relations next to the canonical direction was it observed that two different locative expressions were used, and these are conditions of competition which were associated with the longest production times. However, the observed data on the actual use of the different expressions also suggest that one should be cautious in using applicability ratings for the purpose of selection. Ratings are given under another perspective, and therefore they might measure a different aspect than the one which is effective during production. Although this is not necessarily the case, the data from Franklin, Henkel \& Zangas (1995) as well as our data compared with those from Gapp (1997) demonstrated this difference.

\section{References}

Bryant, D. J., Tversky, B. \& Franklin, N. (1992). Internal and external spatial frameworks for representing described scenes. Journal of Memory and Language, 31, 74-98.

Franklin, N., Henkel, L. A. \& Zangas, T. (1995). Parsing surrounding space into regions. Memory and Cognition, 23, 397-407.

Franklin, N. \& Tversky, B. (1990). Searching imagined environments. Journal of_Experimental Psychology: General, 119, 63-76. 
Gapp, K.-P. (1995). An empirically validated model for computing spatial relations. In I. Wachsmuth, C.-R. Rollinger, and W. Brauer (Ed.), KI-95: Advances in artifical intelligence. Proceedings of the 19th Annual German Conference on Artifical Intelligence (pp. 245-256). Berlin, Heidelberg: Springer.

Gapp, K.-P. (1997). Objektlokalisation: Ein System zur sprachlichen Raumbeschreibung. [The localization of objects: A system for verbal description of locations.] Wiesbaden: Deutscher Universitätsverlag.

Glaser, W. R. \& Glaser, M. O. (1989). Context effects in Stroop-like word and picture processing. Journal of Experimental Psychology: General, 118, 13-42.

Grabowski, J., \& Weiß, P. (1996). Determinanten der Interpretation dimensionaler Lokalisationsäußerungen: Experimente in fünf Sprachen. [Factors that determine the interpretation of dimensional spatial expressions: experiments in five languages.] Sprache \& Kognition, 15, 234-250.

Hayward, W. G. \& Tarr, M. J. (1995). Spatial language and spatial representation. Cognition, $55,39-84$.

Herrmann, T. (1996). Blickpunkte und Blickpunktsequenzen. [Points of view and point of view sequences.] Sprache \& Kognition, 15, 159-177.

Herskovits, A. (1985). Sematics and pragmatics of locative expressions. Cognitive Science, 9, 341-378.

Herskovits, A. (1986). Language and Spatial Cognition. An interdisciplinary Study of the Preposition in English. Cambridge, London: Cambridge University Press.

Klein, W. (1994). Keine Känguruhs zur Linken. [No kangaroos at the left.] In H. J. Kornadt, J. Grabowski \& R. Mangold-Allwinn (Eds.), Sprache und Kognition: Perspektiven moderner Sprachpsychologie (pp. 163-182). Heidelberg: Spektrum.

Levelt, W. J. M. (1989). Speaking: From intention to articulation. Cambridge: MIT Press.

Logan, G. D. (1995). Linguistic and conceptual control of visual spatial attention. Cognitive Pychology, 28, 103-174.

Logan, G. D. \& Sadler, D. D. (1996). A computational analysis of the apprehension of spatial relations. In P. Bloom, M. A. Peterson, L. Nadel \& M. F. Garrett (Eds.), Language and space. Language, speech, and communication (pp. 493-529). Cambridge, MA: MIT Press.

Pechmann, T, Reetz, H., \& Zerbst, D, (1989). Kritik einer Meßmethode: Zur Ungenauigkeit von voicekey Messungen. [The unreliability of voice key measurements.] Sprache \& Kognition, 8, 65-71.

Retz-Schmidt, G. (1988). Various views on spatial prepositions. AI Magazine, 9 (2), 95-105. 